\section{RMD Open}

Rheumatic \&

Musculoskeletal Diseases

\title{
Effects of ibuprofen on gene expression in chondrocytes from patients with osteoarthritis as determined by RNA-Seq
}

\author{
Antti Pemmari (D) , ${ }^{1}$ Lauri Tuure, ${ }^{1}$ Mari Hämäläinen, ${ }^{1}$ Tiina Leppänen, ${ }^{1}$ \\ Teemu Moilanen, ${ }^{1,2}$ Eeva Moilanen ${ }^{1}$
}

To cite: Pemmari A, Tuure L, Hämäläinen $\mathrm{M}$, et al. Effects of ibuprofen on gene expression in chondrocytes from patients with osteoarthritis as determined by RNA-Seq. RMD Open 2021;7:e001657. doi:10.1136/ rmdopen-2021-001657

- Additional supplemental material is published online only. To view, please visit the journal online (http://dx.doi.org/10. 1136/rmdopen-2021-001657).

Received 8 March 2021 Accepted 30 July 2021

\section{Check for updates}

(c) Author(s) (or their employer(s)) 2021. Re-use permitted under CC BY-NC. No commercial re-use. See rights and permissions. Published by BMJ.

${ }^{1}$ The Immunopharmacology Research Group, Faculty of Medicine and Health Technology, Tampere University and Tampere University Hospital, Tampere, Finland

${ }^{2}$ Coxa Hospital for Joint Replacement, Tampere, Finland

Correspondence to Prof Eeva Moilanen; eeva.moilanen@tuni.fi

\section{ABSTRACT}

Non-steroidal anti-inflammatory drugs are a widely used symptomatic treatment in osteoarthritis $(\mathrm{OA})$, but their effects on cartilage remain controversial. We studied the effects of ibuprofen on gene expression in chondrocytes from patients with OA using RNA-Seq. Chondrocytes were isolated from cartilage samples of patients with OA undergoing knee replacement surgery, cultured with ibuprofen, and total mRNA was sequenced. Differentially expressed genes were identified with edgeR using pairwise comparisons. Functional analysis was performed using ingenuity pathway analysis (IPA). Ibuprofen did not induce statistically significant changes in chondrocyte transcriptome when the cells were cultured in the absence of added cytokines. In inflammatory conditions (when the cells were exposed to the OA-related cytokine interleukin (IL)-1ß), 51 genes were upregulated and 42 downregulated by ibuprofen with fold change $>1.5$ in either direction. The upregulated genes included anti-inflammatory factors and genes associated with cell adhesion, while several mediators of inflammation were among the downregulated genes. IPA analysis revealed ibuprofen having modulating effects on inflammation-related pathways such as integrin IL-8, ERK/MAPK and cAMP-mediated signalling pathways. In conclusion, the effects of ibuprofen on primary $\mathrm{OA}$ chondrocyte transcriptome appear to be neutral in normal conditions, but ibuprofen may shift chondrocyte transcriptome towards anti-inflammatory phenotype in inflammatory environments.

\section{INTRODUCTION}

Non-steroidal anti-inflammatory drugs (NSAIDs) are widely used to treat osteoarthritis $(\mathrm{OA})$ pain but there are some concerns on their effects on chondrocyte biology. ${ }^{1}$

$\mathrm{OA}$ is characterised by constant low-grade joint inflammation and transient inflammatory exacerbations. The inflammatory nature of the disease is evidenced by the increased production of proinflammatory cytokines, particularly interleukin-1 $\beta$ (IL-1 $\beta$ ), interleukin-6 (IL-6) and tumour necrosis factor $\alpha$

\section{Key messages}

- The current evidence about the effects of nonsteroidal anti-inflammatory drugs (NSAIDs) on osteoarthritis $(\mathrm{OA})$ cartilage is conflicting.

- We investigated the effects of ibuprofen on gene expression in OA chondrocytes by using RNA-Seq.

- In neutral conditions (in the absence of added inflammatory factors), ibuprofen had no statistically significant effects on gene expression in $\mathrm{OA}$ chondrocytes.

- In inflammatory conditions mimicked by the presence of interleukin (IL)-1 $\beta$, ibuprofen upregulated several anti-inflammatory factors while downregulating inflammatory mediators such as IL-6 and IL23. Ibuprofen also inhibited phosphatase and tensin homolog (PTEN) signalling.

- The findings support the assumption that NSAIDs are safe for cartilage when treating $O A$ pain. They also may shift chondrocyte transcriptome towards an anti-inflammatory phenotype in $0 \mathrm{~A}$ exacerbations.

$(\mathrm{TNF} \alpha)$. They drive the production of catabolic enzymes such as matrix metalloproteinases (MMPs), accelerating joint destruction. ${ }^{2}$

NSAIDs exert their effects by inhibiting the synthesis of prostanoids, particularly prostaglandin $\mathrm{E}_{2}\left(\mathrm{PGE}_{2}\right)$ by cyclo-oxygenase (COX) enzymes. By altering the balance of proinflammatory and anti-inflammatory mediators in the joint, they have been hypothesised to affect OA pathogenesis. These effects, if any, are however controversial, as both potential benefits (eg, alleviation of joint inflammation and reduction of cartilage catabolism) and harms (eg, impairment of cartilage anabolism and accelerated radiographic joint destruction) have been reported. ${ }^{13}$

We carried out a genome-wide expression analysis on the effects of the NSAID ibuprofen on gene expression in OA chondrocytes in 
Table 1 All genes upregulated or downregulated by ibuprofen in the presence of IL-1 $\beta$ with FC $\geq 1.5$ in either direction

\begin{tabular}{|c|c|c|c|c|c|c|}
\hline Gene & Name & Function & Mean (IL1) & Mean (IL1 +ibu) & FC & adj. P \\
\hline PPARG & $\begin{array}{l}\text { Peroxisome proliferator activated receptor } \\
\text { gamma }\end{array}$ & $\begin{array}{l}\text { Carbohydrate and lipid metabolism, } \\
\text { inflammation }\end{array}$ & 0.3 & 0.9 & 2.87 & $5.0 \mathrm{E}-06$ \\
\hline UMODL1 & Uromodulin like 1 & Regulation of apoptosis? & 0.3 & 0.7 & 2.39 & 0.0011 \\
\hline XIRP1 & Xin actin binding repeat containing 1 & Actin binding & 5.7 & 13.4 & 2.38 & $<1.0 \mathrm{E}-06$ \\
\hline DACT1 & $\begin{array}{l}\text { Dishevelled binding antagonist of beta } \\
\text { catenin } 1\end{array}$ & $\begin{array}{l}\text { Regulation of cell cycle and tissue } \\
\text { development }\end{array}$ & 4 & 8.4 & 2.1 & $<1.0 \mathrm{E}-06$ \\
\hline CSF2/GM-CSF & $\begin{array}{l}\text { Colony stimulating factor } 2=\text { Granulocyte- } \\
\text { macrophage colony stimulating factor }\end{array}$ & $\begin{array}{l}\text { Leucocyte differentiation, immune } \\
\text { response }\end{array}$ & 5.2 & 11.2 & 2.09 & $<1.0 \mathrm{E}-06$ \\
\hline PPARGC1B & PPARG coactivator 1 beta & Regulation of transcription & 0.4 & 0.8 & 2.07 & 0.00024 \\
\hline FAM186B & $\begin{array}{l}\text { Family with sequence similarity } 186 \text { member } \\
\text { B }\end{array}$ & $?$ & 0.4 & 0.7 & 1.92 & 0.0035 \\
\hline SOX17 & SRY-box 17 & Cell proliferation, tissue development & 3.5 & 6.8 & 1.91 & $<1.0 \mathrm{E}-06$ \\
\hline MTSS1 & MTSS1, I-BAR domain containing & Cell adhesion & 6.9 & 12.6 & 1.9 & $<1.0 \mathrm{E}-06$ \\
\hline AKAP6 & A-kinase anchoring protein 6 & $\begin{array}{l}\text { Regulation of cell proliferation, cAMP } \\
\text { signalling }\end{array}$ & 2.1 & 3.9 & 1.89 & $<1.0 \mathrm{E}-06$ \\
\hline PDE5A & Phosphodiesterase $5 \mathrm{~A}$ & Regulation of NO signalling & 1.2 & 2.3 & 1.85 & $7.0 \mathrm{E}-06$ \\
\hline RGS2 & Regulator of G protein signalling 2 & Regulation of $\mathrm{G}$ protein signalling & 52.1 & 95.9 & 1.85 & $<1.0 \mathrm{E}-06$ \\
\hline CAMK2A & $\begin{array}{l}\text { Calcium/calmodulin dependent protein } \\
\text { kinase II alpha }\end{array}$ & $\begin{array}{l}\text { Wnt and TGF } \beta \text { signalling, NF- } \mathrm{kB} \\
\text { activation }\end{array}$ & 1.8 & 3.3 & 1.81 & $<1.0 \mathrm{E}-06$ \\
\hline MAP1LC3C & $\begin{array}{l}\text { Microtubule associated protein } 1 \text { light chain } \\
3 \text { gamma }\end{array}$ & Autophagy & 0.7 & 1.2 & 1.79 & $3.3 \mathrm{E}-05$ \\
\hline NRG1 & Neuregulin 1 & Cell differentiation, signal transduction & 0.7 & 1.3 & 1.78 & $5.9 \mathrm{E}-05$ \\
\hline SELE & Selectin E & Inflammation & 41.3 & 73.5 & 1.78 & $<1.0 \mathrm{E}-06$ \\
\hline FCRLA & Fc receptor like $A$ & Immunoglobulin binding & 4.2 & 7.4 & 1.76 & $<1.0 \mathrm{E}-06$ \\
\hline DENND3 & DENN domain containing 3 & Autophagy & 14.6 & 25.3 & 1.75 & $<1.0 \mathrm{E}-06$ \\
\hline FCRLB & Fc receptor like B & Immunoglobulin binding & 0.8 & 1.4 & 1.73 & $3.3 \mathrm{E}-05$ \\
\hline MOXD1 & Monooxygenase DBH like 1 & Monoamine metabolism & 123.1 & 210.3 & 1.72 & $<1.0 \mathrm{E}-06$ \\
\hline SPNS2 & Sphingolipid transporter 2 & Lipid transport & 2.4 & 4.0 & 1.72 & $<1.0 \mathrm{E}-06$ \\
\hline PODXL & Podocalyxin like & Cell adhesion & 11.2 & 19.0 & 1.71 & $<1.0 \mathrm{E}-06$ \\
\hline RP1 & RP1, axonemal microtubule associated & $?$ & 0.4 & 0.6 & 1.67 & 0.023 \\
\hline IDO1 & Indoleamine 2,3-dioxygenase 1 & $\begin{array}{l}\text { Modulation of inflammation and } \\
\text { cartilage development }\end{array}$ & 1.3 & 2.1 & 1.65 & 0.0012 \\
\hline SCUBE3 & $\begin{array}{l}\text { Signal peptide, CUB domain and EGF like } \\
\text { domain containing } 3\end{array}$ & TGF $\beta$ signalling & 42.5 & 72.5 & 1.64 & $<1.0 \mathrm{E}-06$ \\
\hline KCNJ15 & $\begin{array}{l}\text { Potassium voltage-gated channel subfamily } \\
\text { J member } 15\end{array}$ & Potassium transport & 1.3 & 2.2 & 1.63 & $1.0 \mathrm{E}-06$ \\
\hline SERPINE1 & Serpin family E member 1 & Inhibition of proteolysis & 455.9 & 748.6 & 1.62 & $<1.0 \mathrm{E}-06$ \\
\hline PSD2 & Pleckstrin and Sec7 domain containing 2 & $?$ & 0.4 & 0.6 & 1.62 & 0.035 \\
\hline LINGO1 & $\begin{array}{l}\text { Leucine rich repeat and Ig domain } \\
\text { containing } 1\end{array}$ & $?$ & 1.9 & 3.1 & 1.61 & $<1.0 \mathrm{E}-06$ \\
\hline AKNAD1 & AKNA domain containing 1 & $?$ & 0.6 & 1.0 & 1.60 & 0.0048 \\
\hline STRA6 & Stimulated by retinoic acid 6 & Retinol and adipokine binding & 1.9 & 2.9 & 1.59 & 0.00058 \\
\hline ITGAX & Integrin subunit alpha $X$ & Cell adhesion & 11.0 & 17.4 & 1.58 & $<1.0 \mathrm{E}-06$ \\
\hline KCNN3 & $\begin{array}{l}\text { Potassium calcium-activated channel } \\
\text { subfamily } \mathrm{N} \text { member } 3\end{array}$ & Potassium transport & 2.8 & 4.3 & 1.58 & $<1.0 \mathrm{E}-06$ \\
\hline ICAM5 & Intercellular adhesion molecule 5 & Cell adhesion & 4.5 & 7.2 & 1.58 & $<1.0 \mathrm{E}-06$ \\
\hline FGD4 & FYVE, RhoGEF and PH domain containing 4 & Cytoskeleton organisation & 21.9 & 34.0 & 1.57 & $<1.0 \mathrm{E}-06$ \\
\hline KCNN4 & $\begin{array}{l}\text { Potassium calcium-activated channel } \\
\text { subfamily } \mathrm{N} \text { member } 4\end{array}$ & Potassium transport & 6.4 & 10.0 & 1.57 & $<1.0 \mathrm{E}-06$ \\
\hline LRRC55 & Leucine rich repeat containing 55 & Potassium transport & 0.8 & 1.3 & 1.56 & 0.0013 \\
\hline CXCR3 & C-X-C motif chemokine receptor 3 & Inflammation & 0.7 & 1.1 & 1.55 & 0.017 \\
\hline CD24 & CD24 molecule & $\begin{array}{l}\text { Wnt and MAPK signalling, regulation } \\
\text { of inflammation }\end{array}$ & 5.4 & 8.5 & 1.55 & $<1.0 \mathrm{E}-06$ \\
\hline FGR & $\begin{array}{l}\text { FGR proto-oncogene, Src family tyrosine } \\
\text { kinase }\end{array}$ & $\begin{array}{l}\text { PI3K-Akt signalling, regulation of } \\
\text { inflammation }\end{array}$ & 3.4 & 5.2 & 1.54 & $<1.0 \mathrm{E}-06$ \\
\hline
\end{tabular}


Table 1 Continued

\begin{tabular}{|c|c|c|c|c|c|c|}
\hline Gene & Name & Function & Mean (IL1) & Mean (IL1 +ibu) & FC & adj. $P$ \\
\hline PEG10 & Paternally expressed 10 & Inhibition of TGF $\beta$ signalling & 23.9 & 36.4 & 1.54 & $<1.0 \mathrm{E}-06$ \\
\hline SIGLEC15 & Sialic acid binding Ig like lectin 15 & Regulation of bone resorbtion & 1.6 & 2.4 & 1.54 & 0.00024 \\
\hline CPNE2 & Copine 2 & Bone erosion & 18.7 & 28.9 & 1.54 & $<1.0 \mathrm{E}-06$ \\
\hline WNK4 & WNK lysine deficient protein kinase 4 & Ion transport & 4.6 & 7.0 & 1.53 & $<1.0 \mathrm{E}-06$ \\
\hline RTL3 & Retrotransposon Gag like 3 & Regulation of collagen production & 3.3 & 5.0 & 1.53 & $<1.0 \mathrm{E}-06$ \\
\hline RGS3 & Regulator of G protein signalling 3 & Inhibition of MAPK signalling & 65.1 & 99.2 & 1.52 & $<1.0 \mathrm{E}-06$ \\
\hline $\mathrm{AOC2}$ & Amine oxidase, copper containing 2 & Amine metabolism & 68.0 & 102.3 & 1.51 & $<1.0 \mathrm{E}-06$ \\
\hline IL10RA & Interleukin 10 receptor subunit alpha & Regulation of inflammation & 1.0 & 1.6 & 1.51 & 0.0018 \\
\hline RGS16 & Regulator of G protein signalling 16 & $?$ & 60.1 & 90.0 & 1.51 & $<1.0 \mathrm{E}-06$ \\
\hline $\mathrm{PCDH} 17$ & Protocadherin 17 & Cell adhesion & 0.9 & 1.4 & 1.51 & 0.028 \\
\hline GPR158 & G protein-coupled receptor 158 & $?$ & 1.3 & 1.9 & 1.50 & 0.00017 \\
\hline IL23A & Interleukin 23 subunit alpha & Inflammation & 15.2 & 4.7 & -3.24 & $<1.0 \mathrm{E}-06$ \\
\hline HAS1 & Hyaluronan synthase 1 & Extracellular matrix production & 0.8 & 0.3 & -2.77 & $<1.0 \mathrm{E}-06$ \\
\hline IGFBP4 & Insulin-like growth factor binding protein 4 & Cell proliferation and metabolism & 213.8 & 79.7 & -2.73 & $<1.0 \mathrm{E}-06$ \\
\hline IL6 & Interleukin 6 & Inflammation & 958.4 & 403.8 & -2.49 & $<1.0 \mathrm{E}-06$ \\
\hline PDE3A & Phosphodiesterase $3 \mathrm{~A}$ & Lipid metabolism & 0.9 & 0.3 & -2.48 & 0.00013 \\
\hline STAT4 & $\begin{array}{l}\text { Signal transducer and activator of } \\
\text { transcription } 4\end{array}$ & $\begin{array}{l}\text { Inflammation, regulation of cell } \\
\text { proliferation }\end{array}$ & 2.5 & 1.0 & -2.36 & $<1.0 \mathrm{E}-06$ \\
\hline PCSK1 & Proprotein convertase subtilisin/kexin type 1 & Metabolism & 7.2 & 3.2 & -2.19 & $<1.0 \mathrm{E}-06$ \\
\hline ADAMTS6 & $\begin{array}{l}\text { ADAM metallopeptidase with } \\
\text { thrombospondin type } 1 \text { motif } 6\end{array}$ & Extracellular matrix catabolism & 10.5 & 4.9 & -2.18 & $<1.0 \mathrm{E}-06$ \\
\hline HAL & Histidine ammonia-lyase & Histidine catabolism & 1.7 & 0.8 & -2.12 & $<1.0 \mathrm{E}-06$ \\
\hline DNAH17 & Dynein axonemal heavy chain 17 & Cytoskeleton component & 1.0 & 0.5 & -2.06 & $2.00 \mathrm{E}-06$ \\
\hline CSF3 & Colony stimulating factor 3 & $\begin{array}{l}\text { Inflammation, regulation of cell } \\
\text { proliferation }\end{array}$ & 19.8 & 9.9 & -2.02 & $<1.0 \mathrm{E}-06$ \\
\hline AREG & Amphiregulin & $\begin{array}{l}\text { EGF signalling, regulation of cell } \\
\text { proliferation }\end{array}$ & 2.3 & 1.2 & -2.01 & $<1.0 \mathrm{E}-06$ \\
\hline CA12 & Carbonic anhydrase 12 & $\begin{array}{l}\text { Acidity regulation, Regulation of } \\
\text { proliferation }\end{array}$ & 20.9 & 10.5 & -2.00 & $<1.0 \mathrm{E}-06$ \\
\hline INSC & Inscuteable homolog (Drosophila) & Cell differentiation & 0.6 & 0.3 & -1.98 & 0.0011 \\
\hline KCNE5 & $\begin{array}{l}\text { Potassium voltage-gated channel subfamily } \\
\text { E regulatory subunit } 5\end{array}$ & Regulation of potassium transport & 1.3 & 0.6 & -1.94 & $6.00 \mathrm{E}-06$ \\
\hline LDB2 & LIM domain binding 2 & Regulation of transcription & 0.5 & 0.3 & -1.92 & 0.005098 \\
\hline DOK6 & Docking protein 6 & $?$ & 0.9 & 0.5 & -1.80 & 0.000598 \\
\hline DAW1 & Dynein assembly factor with WD repeats 1 & Dynein assembly & 0.9 & 0.5 & -1.78 & 0.000565 \\
\hline TMEM71 & Transmembrane protein 71 & $?$ & 1.8 & 1.0 & -1.77 & $2.00 E-06$ \\
\hline MAMSTR & $\begin{array}{l}\text { MEF2 activating motif and SAP domain } \\
\text { containing transcriptional regulator }\end{array}$ & Regulation of transcription & 0.5 & 0.3 & -1.72 & 0.021819 \\
\hline KNDC1 & $\begin{array}{l}\text { Kinase non-catalytic C-lobe domain } \\
\text { containing } 1\end{array}$ & $?$ & 0.8 & 0.5 & -1.70 & 0.002773 \\
\hline EFHC2 & EF-hand domain containing 2 & Cell proliferation & 0.8 & 0.5 & -1.69 & 0.004747 \\
\hline MEXЗА & Mex-3 RNA binding family member A & PI3K-Akt signalling & 0.9 & 0.5 & -1.69 & 0.001905 \\
\hline TGFBI & Transforming growth factor beta induced & $\begin{array}{l}\text { ECM organisation, chondrocyte } \\
\text { differentiation }\end{array}$ & 127.8 & 80.7 & -1.64 & $<1.0 \mathrm{E}-06$ \\
\hline C3AR1 & Complement C3a receptor 1 & Inflammation & 3.5 & 2.2 & -1.63 & $<1.0 \mathrm{E}-06$ \\
\hline EFEMP1 & $\begin{array}{l}\text { EGF containing fibulin like extracellular } \\
\text { matrix protein } 1\end{array}$ & $\begin{array}{l}\text { Inhibition of chondrocyte } \\
\text { differentiation }\end{array}$ & 72.6 & 45.4 & -1.63 & $<1.0 \mathrm{E}-06$ \\
\hline NAMPT & $\begin{array}{l}\text { Nicotinamide phosphoribosyltransferase / } \\
\text { visfatin }\end{array}$ & Cartilage catabolism & 596.1 & 368.8 & -1.60 & $<1.0 \mathrm{E}-06$ \\
\hline FOXF1 & Forkhead box F1 & Morphogenesis & 1.2 & 0.8 & -1.60 & 0.000928 \\
\hline AVPI1 & Arginine vasopressin induced 1 & MAPK signalling & 39.7 & 24.8 & -1.60 & $<1.0 \mathrm{E}-06$ \\
\hline SEMA3A & Semaphorin $3 \mathrm{~A}$ & $\begin{array}{l}\text { Regulation of inflammation and } \\
\text { apoptosis }\end{array}$ & 98.0 & 61.6 & -1.59 & $<1.0 \mathrm{E}-06$ \\
\hline STC1 & Stanniocalcin 1 & Regulation of cartilage development & 2.0 & 1.3 & -1.59 & 0.002967 \\
\hline
\end{tabular}




\begin{tabular}{|c|c|c|c|c|c|c|}
\hline Gene & Name & Function & Mean (IL1) & Mean (IL1 +ibu) & FC & adj. P \\
\hline TSKU & Tsukushi, small leucine rich proteoglycan & $?$ & 14.9 & 9.4 & -1.58 & $<1.0 \mathrm{E}-06$ \\
\hline SMOC1 & SPARC related modular calcium binding 1 & ECM organisation & 199.0 & 126.1 & -1.57 & $<1.0 \mathrm{E}-06$ \\
\hline ARAP2 & $\begin{array}{l}\text { ArfGAP with RhoGAP domain, ankyrin } \\
\text { repeat and PH domain } 2\end{array}$ & $\begin{array}{l}\text { Cell adhesion, inhibition of Akt } \\
\text { signalling }\end{array}$ & 12.5 & 8.0 & -1.56 & $<1.0 \mathrm{E}-06$ \\
\hline BEND5 & BEN domain containing 5 & Negative regulation of transcription & 0.9 & 0.6 & -1.54 & 0.01673 \\
\hline EPB41L3 & $\begin{array}{l}\text { Erythrocyte membrane protein band } 4.1 \\
\text { like } 3\end{array}$ & Cortical cytoskeleton organisation & 34.6 & 22.5 & -1.53 & $<1.0 \mathrm{E}-06$ \\
\hline EPB41L4B & $\begin{array}{l}\text { Erythrocyte membrane protein band } 4.1 \\
\text { like } 4 B\end{array}$ & $\begin{array}{l}\text { Regulation of cell adhesion and gene } \\
\text { expression }\end{array}$ & 2.8 & 1.8 & -1.53 & 0.000106 \\
\hline ACSL4 & $\begin{array}{l}\text { Acyl-CoA synthetase long-chain family } \\
\text { member } 4\end{array}$ & Lipid metabolism & 428.1 & 280.7 & -1.52 & $<1.0 \mathrm{E}-06$ \\
\hline NR4A2 & $\begin{array}{l}\text { Nuclear receptor subfamily } 4 \text { group A } \\
\text { member } 2\end{array}$ & Wnt signalling, regulation of apoptosis & 19.8 & 13.1 & -1.51 & $<1.0 \mathrm{E}-06$ \\
\hline PID1 & $\begin{array}{l}\text { Phosphotyrosine interaction domain } \\
\text { containing } 1\end{array}$ & Oxidative metabolism & 285.1 & 190.6 & -1.51 & $<1.0 \mathrm{E}-06$ \\
\hline RSPO3 & R-spondin 3 & Wnt signalling, morphogenesis & 82.9 & 53.8 & -1.50 & $<1.0 \mathrm{E}-06$ \\
\hline
\end{tabular}

Red $=$ upregulated genes; blue $=$ downregulated genes .

adj. P, False discovery rate (FDR) -adjusted $\mathrm{P}$ value; IL, interleukin; Mean, trimmed mean of M-values (TMM) normalized counts.

normal and inflammatory conditions in vitro by using RNA-Seq.

\section{METHODS}

Cartilage samples were obtained from 10 patients with OA (mean age 67 years (SEM 3.8 years), 8 females, Kellgren-Lawrence grade 3.7 (SEM 0.15)) undergoing knee replacement surgery in Coxa Hospital for Joint Replacement, Tampere, Finland.

Chondrocytes were isolated by enzyme digestion and seeded on 24-well plates for 24 hours. Thereafter the experiments were started, and the cells were cultured either alone, with ibuprofen $(10 \mu \mathrm{M})$, with IL-1 $\beta(100 \mathrm{pg} /$ $\mathrm{mL}$ ), or with a combination of ibuprofen and IL-1 $\beta$ for 24 hours. Cell culture, RNA sequencing, RT-PCR and data analysis are described in online supplemental data S1.

\section{RESULTS}

\section{The effects of ibuprofen on $\mathrm{OA}$ chondrocytes in neutral conditions}

In the absence of exogenous cytokines, no genes were found to be differentially expressed between chondrocytes cultured with or without ibuprofen when the results were adjusted by false discovery rate.

\section{The effects of ibuprofen on $\mathrm{OA}$ chondrocytes in inflammatory conditions}

In inflammatory conditions (ie, in the presence of the OA-related cytokine IL-1 $\beta$ ), ibuprofen induced the upregulation of 51 genes while 42 were downregulated in a statistically significant manner with a fold change $>1.5$ into either direction (table 1 ). All differentially expressed genes are listed in online supplemental tables S2 and S3.

The upregulated genes included anti-inflammatory factors such as peroxisome proliferator-activated receptor gamma (PPARG) and its coactivator PPARGC1B as well as IL-10 receptor subunit alpha. In addition, some genes associated with inflammation, including C-X-C motif chemokine receptor 3 (CXCR3), selectin $\mathrm{E}(S E L E)$ and granulocyte-macrophage colony stimulating factor (CSF2/GM-CSF) were also upregulated (table 1).

On the other hand, several mediators of inflammation (such as IL23A, IL6 and NAMPT (nicotinamide phosphoribosyltransferase aka visfatin)) were downregulated, as was the catabolic enzyme ADAMTS6 (ADAM metallopeptidase with thrombospondin type 1 motif 6 ). Insulin-like growth factor-binding protein 4 (IGFBP4), which sequesters IGF and regulates chondrocyte proliferation, ${ }^{4}$ was also downregulated. Hyaluronan synthase 1 (HAS1) and stanniocalcin-1 (STC1), previously shown to be upregulated in inflamed OA synovium, ${ }^{5}$ were also downregulated by ibuprofen (table 1).

Differential expression of selected inflammation and cartilage-related genes (PPARG, PPARGC1B, CSF2, IL23, HAS1, IGFBP4, ADAMTS6 and ILO) was confirmed with RT-PCR using chondrocytes from a different set of 10 patients (online supplemental figure S4). As expected, IL-1 $\beta$ was shown to strongly increase the synthesis of prostanoids, and this increase was inhibited by ibuprofen (online supplemental figure S5).

When all genes affected by ibuprofen in a statistically significant manner in the presence of IL-1 $\beta$ were analysed with ingenuity pathway analysis (IPA), activated canonical pathways included several associated with inflammation and cell adhesion such as IL-8, integrin, ERK/MAPK and cAMP-mediated signalling pathways (table 2). Conversely, phosphatase and tensin homolog (PTEN) signalling was inhibited (table 2). Differentially expressed genes included in the significantly activated/ inhibited pathways are listed in online supplemental table S6. 
Table 2 Canonical IPA pathways significantly upregulated or downregulated ( $z$-score $\geq 2.5$ or $\leq-2.5$ ) by ibuprofen in the presence of IL-1 $\beta$

\begin{tabular}{|c|c|c|}
\hline Canonical pathway & adj. P & z-score \\
\hline Integrin signalling & 4.37E-08 & 4.95 \\
\hline Actin cytoskeleton signalling & 0.0022 & 4.24 \\
\hline PI3K signalling in B lymphocytes & 0.00032 & 3.44 \\
\hline $\begin{array}{l}\text { Agrin Interactions at } \\
\text { neuromuscular junction }\end{array}$ & 0.0037 & 3.32 \\
\hline IL-8 signalling & $7.08 \mathrm{E}-07$ & 3.29 \\
\hline ERK5 signalling & 0.0083 & 3.16 \\
\hline Glioblastoma multiforme signalling & $1.32 \mathrm{E}-06$ & 3.14 \\
\hline Paxillin signalling & 4.27E-06 & 3.05 \\
\hline ErbB2-ErbB3 signalling & 0.029 & 3.00 \\
\hline $\begin{array}{l}\text { FcyRIIB signalling in B } \\
\text { lymphocytes }\end{array}$ & 0.025 & 3.00 \\
\hline Renal cell carcinoma signalling & 0.0016 & 3.00 \\
\hline Bladder cancer signalling & $6.31 \mathrm{E}-06$ & 3.00 \\
\hline 14-3-3-mediated signalling & 0.0058 & 2.89 \\
\hline PKC $\theta$ signalling in T lymphocytes & 0.030 & 2.84 \\
\hline Calcium signalling & 0.0083 & 2.84 \\
\hline Thrombin signalling & 0.0025 & 2.83 \\
\hline CREB signalling in neurons & 0.0019 & 2.83 \\
\hline HGF signalling & $1.15 \mathrm{E}-06$ & 2.83 \\
\hline $\begin{array}{l}\text { Non-small cell lung cancer } \\
\text { signalling }\end{array}$ & 0.0029 & 2.83 \\
\hline$\alpha$-Adrenergic signalling & 5.37E-06 & 2.71 \\
\hline Endothelin-1 signalling & 0.0098 & 2.68 \\
\hline $\begin{array}{l}\text { Mouse embryonic stem cell } \\
\text { pluripotency }\end{array}$ & 0.0052 & 2.67 \\
\hline NF- $\kappa B$ activation by viruses & 0.00089 & 2.67 \\
\hline Macropinocytosis signalling & $4.27 \mathrm{E}-07$ & 2.67 \\
\hline CXCR4 signalling & 0.0048 & 2.67 \\
\hline p70S6K signalling & 0.0026 & 2.67 \\
\hline cAMP-mediated signalling & 0.0034 & 2.56 \\
\hline ErbB4 signalling & 0.014 & 2.53 \\
\hline Chemokine signalling & 0.013 & 2.53 \\
\hline $\begin{array}{l}\text { Actin nucleation by ARP-WASP } \\
\text { complex }\end{array}$ & 0.00078 & 2.53 \\
\hline $\begin{array}{l}\text { Regulation of cellular mechanics } \\
\text { by calpain protease }\end{array}$ & $5.25 \mathrm{E}-05$ & 2.53 \\
\hline Synaptic long-term potentiation & 0.00011 & 2.52 \\
\hline Cardiac hypertrophy signalling & 0.00015 & 2.50 \\
\hline ERK/MAPK signalling & $1.91 \mathrm{E}-05$ & 2.50 \\
\hline fMLP signalling in neutrophils & 0.0012 & 2.50 \\
\hline PAK signalling & 0.00013 & 2.50 \\
\hline Rac signalling & 0.026 & 2.50 \\
\hline IL-3 signalling & 0.0018 & 2.50 \\
\hline Acute myeloid leukaemia signalling & 0.0017 & 2.50 \\
\hline
\end{tabular}

Continued

\begin{tabular}{llc} 
Table 2 Continued & & \\
\hline Canonical pathway & adj. P & z-score \\
\hline Telomerase signalling & 0.0011 & $\mathbf{2 . 5 0}$ \\
Wnt/Ca+pathway & $5.25 \mathrm{E}-05$ & $\mathbf{2 . 5 0}$ \\
PTEN signalling & 0.00087 & $\mathbf{- 2 . 6 7}$
\end{tabular}

adj.P, False discovery rate (FDR) -adjusted $P$ value; CREB, CAMP response element-binding protein; IL-1 $\beta$, interleukin $1 \beta$; IPA, ingenuity pathway analysis.

Among the genes with FC $>1.5$ in either direction, STRING analysis identified IL6 (which was downregulated by ibuprofen) as a central node in the interaction network (figure 1). Other genes occupying central places include PPARG, granulocyte-macrophage colonystimulating factor and selectin E (PPARG, CSF2 and SELE respectively, all upregulated by ibuprofen).

\section{DISCUSSION}

Ibuprofen did not have any significant effects on gene expression in primary OA chondrocytes cultured in the absence of added cytokines. This implies that ibuprofen has a neutral effect on chondrocyte transcriptome in non-inflamed joints. In cells treated with IL-1 $\beta$, ibuprofen regulated the expression of both proinflammatory and anti-inflammatory factors and seemed to shift the balance to favour the latter.

Ibuprofen is a widely used non-selective NSAID. Like other NSAIDs, it exerts its effects by inhibiting prostanoid, particularly $\mathrm{PGE}_{2}$, synthesis by COX-1 and COX-2 enzymes. In addition to their role as mediators of a pain, prostanoids such as $\mathrm{PGE}_{2}$ mediate various inflammatory responses. Prostanoids have also been implicated in the pathogenesis OA by affecting cartilage matrix integrity and proteoglycan degradation as well as chondrocyte dedifferentiation and apoptosis. ${ }^{16}$ Cellular effects of prostanoids are mediated through G-protein coupled receptors; many prostaglandin receptor subtypes, particularly $\mathrm{DP}_{1}, \mathrm{EP}_{2}, \mathrm{EP}_{4}$ and $\mathrm{IP}^{7}$ activate adenylate cyclase leading to increased intracellular levels of the multifunctional second messenger cAMP. By activating protein kinase $\mathrm{A}$ and transcription factors such as cAMP response element-binding protein, cAMP also regulates the expression of a number of genes. ${ }^{8}$ This pathway offers a possible prostanoid-dependent mechanism for the changes in gene expression seen in the present study. In addition, the IPA analysis showed that ibuprofen regulates several other inflammatory pathways which may mediate its effects on chondrocyte transcriptome by prostanoid dependent or independent manner.

In our data, ibuprofen increased the expression of $P P A R G$ and its coactivator 1 beta (PPARGC1B). PPARG expression has been shown to be downregulated in OA cartilage, ${ }^{9}$ and PPARG may affect the pathogenesis of OA by suppressing joint inflammation, downregulating the production of catabolic enzymes and inhibiting 


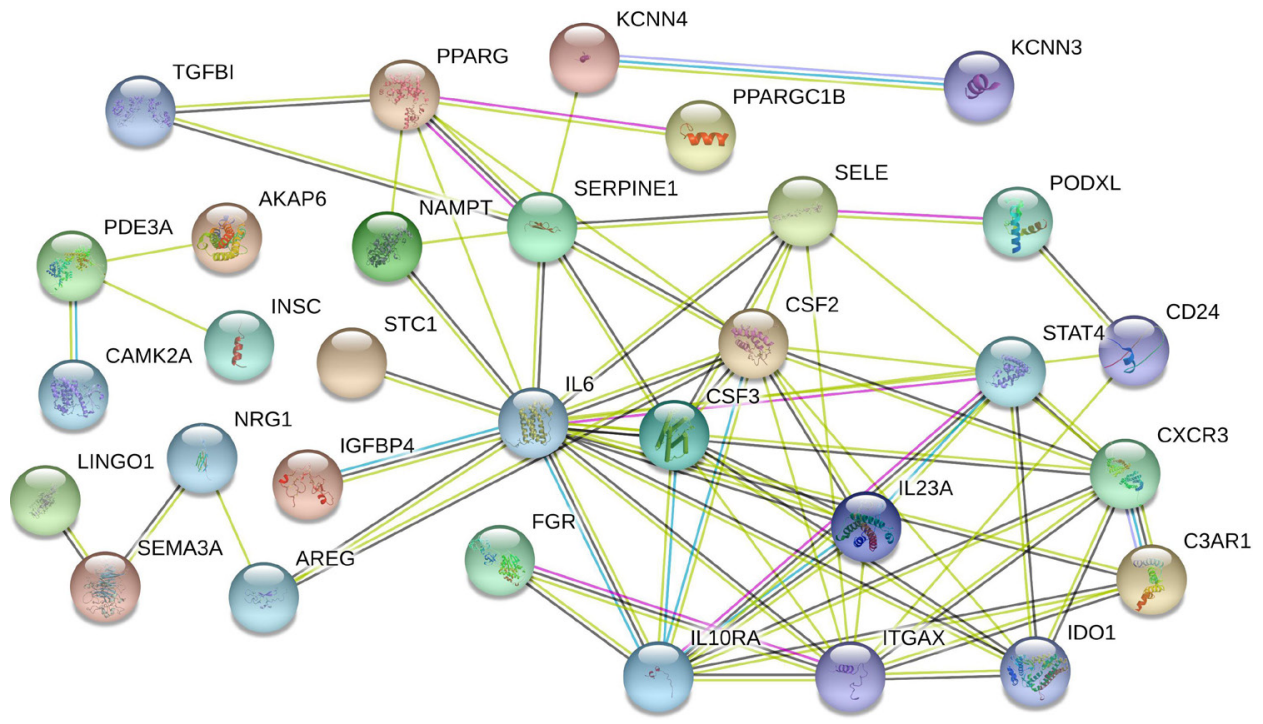

Figure 1 Interactions among the genes that were upregulated or downregulated by ibuprofen with an FC 1.5 or greater (in either direction) in IL-1 $\beta$-treated cells. Genes with no identified interactions are excluded from the graph. Colours of the edges: green=activation, blue=binding, black=chemical reaction, red=inhibition, violet=catalysis, pink=posttranslational modification, yellow=transcriptional regulation, grey=other interaction.

chondrocyte apoptosis. ${ }^{10}$ Induction of some proinflammatory factors such as CSF2/GM-CSF by ibuprofen can be regarded as a potentially deleterious effect, as CM-CSF has been shown to promote OA development and pain. ${ }^{11}$ To our knowledge, this is the first study linking NSAIDs to GM-CSF production in chondrocytes.

IL6 and IL23A as well as ADAMTS6 (ADAM metallopeptidase with thrombospondin type 1 motif 6 ) are examples of proinflammatory/catabolic factors that were suppressed by ibuprofen. Ibuprofen downregulated also hyaluronan synthase 1 (HAS1) and stanniocalcin-1 (STC1) both of which have been shown to be upregulated in inflamed OA joints. ${ }^{5}$ These data suggest that ibuprofen can, to some extent, 'normalise' the phenotype of OA tissue under inflammatory conditions. Notably, IL23A was the most strongly downregulated gene in our data. The potential local roles of this proinflammatory cytokine in OA cartilage appear relatively understudied, but its serum levels in patients with OA have been found to be higher compared with controls. ${ }^{12}$ IL-6 is considered a central proinflammatory mediator in OA. ${ }^{13}$ HAS1 is one of the three principal enzymes participating in the synthesis of hyaluronan, a central extracellular matrix (ECM) component. It may also promote inflammation by producing pericellular, monocyte-attracting hyaluronan coats. ${ }^{14}$ STC1 is a calcium-regulating and phosphateregulating protein whose effects on cartilage appear to be complex. It may inhibit cartilage development, ${ }^{15}$ but its expression in synovial cells has also been linked to slower OA progression. ${ }^{16}$

Integrin signalling was the IPA pathway most strongly activated by ibuprofen. This is interesting, as dysregulated integrin signalling has been implicated in OA pathogenesis. ${ }^{17}$ Other significantly upregulated pathways include several linked to inflammation (such as
IL-8, NF-אB and MAPK/ERK signalling). Looking at the specific genes included in these pathways and affected by ibuprofen (online supplemental table S6) reveals that these can be mostly considered negative feedback genes rather than the major proinflammatory mediators/effectors of these pathways. Examples include several integrins (ITGAM, ITGAX, ITGB2, ITGB3 and ITGB5) in the IL-8 and NF-אB pathways, growth factors and their receptors (VEGFA, VEGFC, HBEGF and FGFR3) in IL-8 signalling as well as anti-inflammatory MAPK phosphatases and PPAR pathway constituents (DUSP1, DUSP2, DUSP4, PRKAR1A, PRKAR1B, PRKAR2B and PPARG) in MAPK/ ERK signalling.

Intriguingly, PTEN signalling was inhibited by ibuprofen. PTEN is a modulator of phosphoinositide 3-kinase/Akt (PI3K/Akt) signalling with various potential effects including promotion of apoptosis, regulation of cell adhesion and inhibition of cell proliferation. PTEN is upregulated in OA chondrocytes, where it inhibits the production of ECM components, ${ }^{18}$ and interventions that inhibit PTEN slow the development of osteoarthritic changes in cartilage. ${ }^{19}$ To our knowledge, PTEN has not previously been linked to NSAIDs in cartilage.

Previous studies have investigated the effects of NSAIDs and COX-2 selective inhibitors on cartilage/ synovial explants. ${ }^{620}$ Both prostaglandin-mediated and prostaglandin-independent effects have been observed; these include, for example, inhibition of chondrocyte apoptosis, reduction of nitric oxide synthesis as well as reduced production of catabolic MMPs on IL-1 $\beta$ stimulation. ${ }^{1}$ Our study expands these results by investigating the whole transcriptome of ibuprofen-treated OA chondrocytes and provides a starting point for future studies.

In conclusion, ibuprofen alone had no significant effects on gene expression in chondrocytes supporting 
cartilage safety of COX inhibitors in the treatment of OA pain. When used in a setting of joint inflammation, ibuprofen seems to shift chondrocyte transcriptome towards an anti-inflammatory phenotype.

Acknowledgements We thank research coordinator Heli Kupari for her assistance with the cartilage samples. We are also grateful to Ms. Meiju Kukkonen and Mrs. Salla Hietakangas for excellent technical assistance, as well as to Mrs. Heli Määttä for great secretarial help.

Contributors Conceptualisation, methodology: all authors. Formal analysis: AP, TM, EM. Investigation: AP, LT, TL, MH. Writing: AP, EM. Visualisation: AP. Supervision and funding: EM.

Funding This study was supported by grants from Finnish Society of Rheumatology and the Competitive State Research Financing of the Expert Responsibility Area of Tampere University Hospital.

Disclaimer The funders had no role in study design, data collection and analysis, decision to publish, or preparation of the manuscript.

Competing interests None declared.

Patient consent for publication Not required.

Ethics approval The study was approved by the Ethics Committee of Tampere University Hospital, Tampere, Finland (decision reference ETL R16076), and performed in accordance with the Declaration of Helsinki. Written informed consent was obtained from the patients.

Provenance and peer review Not commissioned; externally peer reviewed.

Open access This is an open access article distributed in accordance with the Creative Commons Attribution Non Commercial (CC BY-NC 4.0) license, which permits others to distribute, remix, adapt, build upon this work non-commercially, and license their derivative works on different terms, provided the original work is properly cited, appropriate credit is given, any changes made indicated, and the use is non-commercial. See: http://creativecommons.org/licenses/by-nc/4.0/.

ORCID iD

Antti Pemmari http://orcid.org/0000-0002-8187-1216

\section{REFERENCES}

1 Nakata K, Hanai T, Take Y, et al. Disease-Modifying effects of COX-2 selective inhibitors and non-selective NSAIDs in osteoarthritis: a systematic review. Osteoarthritis Cartilage 2018;26:1263-73.

2 Berenbaum F. Osteoarthritis as an inflammatory disease (osteoarthritis is not osteoarthrosis!). Osteoarthritis Cartilage 2013;21:16-21.

3 Ding C. Do NSAIDs affect the progression of osteoarthritis? Inflammation 2002;26:139-42.
4 Gruber HE, Hoelscher GL, Ingram JA, et al. Human annulus cells regulate PAPP-A and IGFBP-4 expression, and thereby insulin-like growth factor bioavailability, in response to proinflammatory cytokine exposure in vitro. Connect Tissue Res 2013:54:432-8.

5 Lambert C, Dubuc J-E, Montell E, et al. Gene expression pattern of cells from inflamed and normal areas of osteoarthritis synovial membrane. Arthritis Rheumatol 2014;66:960-8.

6 Hardy MM, Seibert K, Manning PT, et al. Cyclooxygenase 2-dependent prostaglandin E2 modulates cartilage proteoglycan degradation in human osteoarthritis explants. Arthritis Rheum 2002;46:1789-803.

7 Clapp L, Giembycz M, Heinemann A. Prostanoid receptors (version 2020.2) in the IUPHAR/BPS guide to pharmacology database. IUPHAR/BPS Guide to Pharmacology 2020;2.

8 Raker VK, Becker C, Steinbrink K. The cAMP pathway as therapeutic target in autoimmune and inflammatory diseases. Front Immunol 2016;7:123.

9 Afif $\mathrm{H}$, Benderdour M, Mfuna-Endam L, et al. Peroxisome proliferator-activated receptor gamma1 expression is diminished in human osteoarthritic cartilage and is downregulated by interleukin1 beta in articular chondrocytes. Arthritis Res Ther 2007;9:R31.

10 Fahmi H, Martel-Pelletier J, Pelletier J-P, et al. Peroxisome proliferator-activated receptor gamma in osteoarthritis. Mod Rheumatol 2011;21:1-9.

11 Conaghan PG, Cook AD, Hamilton JA, et al. Therapeutic options for targeting inflammatory osteoarthritis pain. Nat Rev Rheumato 2019;15:355-63.

12 Askari A, Naghizadeh MM, Homayounfar R, et al. Increased serum levels of IL-17A and IL-23 are associated with decreased vitamin D3 and increased pain in osteoarthritis. PLoS One 2016;11:e0164757.

13 Wojdasiewicz P, Poniatowski kukasz A, Szukiewicz D. The role of inflammatory and anti-inflammatory cytokines in the pathogenesis of osteoarthritis. Mediators Inflamm 2014;2014:1-19.

14 Meran S, Martin J, Luo DD, et al. Interleukin-1 $\beta$ induces hyaluronan and CD44-dependent cell protrusions that facilitate fibroblastmonocyte binding. Am J Pathol 2013;182:2223-40.

15 Wu S, Yoshiko Y, De Luca F. Stanniocalcin 1 acts as a paracrine regulator of growth plate chondrogenesis. J Biol Chem 2006;281:5120-7.

16 Wu Y, Li Z, Jia W, et al. Upregulation of stanniocalcin-1 inhibits the development of osteoarthritis by inhibiting survival and inflammation of fibroblast-like synovial cells. J Cell Biochem 2019;120:9768-80.

17 Peters JH, Loredo GA, Benton HP. Is osteoarthritis a 'fibronectinintegrin imbalance disorder'? Osteoarthritis Cartilage 2002;10:831-5.

18 Iwasa K, Hayashi S, Fujishiro T, et al. PTEN regulates matrix synthesis in adult human chondrocytes under oxidative stress. $J$ Orthop Res 2014;32:231-7.

19 Chen Y, Zhang L, Li E, et al. Long-Chain non-coding RNA HOTAIR promotes the progression of osteoarthritis via sponging miR-20b/ PTEN axis. Life Sci 2020;253:117685.

20 Williams A, Smith JR, Allaway D, et al. Carprofen inhibits the release of matrix metalloproteinases 1,3 , and 13 in the secretome of an explant model of articular cartilage stimulated with interleukin $1 \beta$. Arthritis Res Ther 2013;15:R223. 